\title{
Mesure de la tension artérielle: expérience personnelle et quotidien des médecins
}

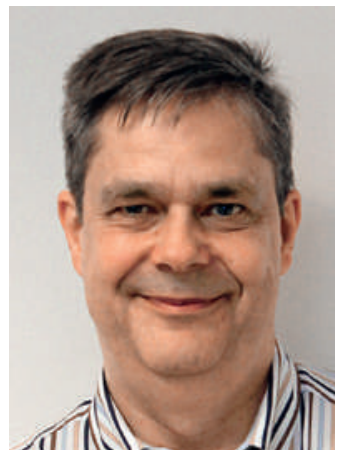

Eberhard Wolff
* PD Dr ès sc. soc. Eberhard Wolff est licencié en sciences humaines, historien de la médecine et membre de la rédaction Histoire de la médecine du Bulletin des médecins suisses.
«Je prends rapidement votre tension.» Simple routine dans les professions médicales, lors des "petits examens». N'importe quel professionnel vous le dira: c'est simple, rapide et les résultats sont immédiats. Ces mesures ont montré chez moi, ces derniers temps, une légère hypertonie. Simple routine dans le quotidien des médecins.

N'importe quel professionnel vous le dira: des mesures ponctuelles chez le médecin ne veulent pas dire grand-chose et il faut garder un certain recul. La nervosité générée par la situation peut provoquer des valeurs erronées. Mon médecin m'a confié un appareil mesurant la tension sur $24 \mathrm{~h}$. La courbe affichée n'avait rien à envier à la chaîne des Churfirsten. N'importe quel professionnel vous le dira: la tension artérielle est versatile et dépend de nombreux facteurs. Mais quand, en un quart d'heure, les valeurs augmentent ou diminuent de 30 , voire $40 \mathrm{~mm} \mathrm{Hg}$, alors que j'étais calme ce jour-là, je suis en droit de me demander si même un appareil professionnel ne peut pas se tromper.

Pourtant, au vu de la courbe et de la moyenne, le diagnostic de mon médecin est tombé: tension élevée, à surveiller. Il m'a recommandé un autotensiomètre. Selon lui, les produits Migros à fixer au poignet ou au bras n'étaient pas mal. Nous en avions un à la maison, à fixer au poignet. Mesurer la tension artérielle est très simple. Le premier jour, l'appareil afficha les valeurs les plus diverses, et au repos des chiffres dignes de ceux d'un jeu d'ordinateur juste avant que le zombie n'explose. Pourquoi? Mauvaise qualité de l'appareil? Mauvaise utilisation? Mauvaise pente de ma pression artérielle? Mesurer la tension artérielle est pourtant simple! J'avoue avoir fait une erreur: j'aurais dû tourner l'appareil à $180^{\circ}$ pour prendre mon pouls. Un professionnel devrait le savoir. Au moins, les valeurs de zombie avaient disparu.

Mais même après, des écarts de la pression systolique entre 140 et $180 \mathrm{~mm} \mathrm{Hg}$ en une minute n'étaient pas rares. La pression artérielle serait-elle comme une Ferrari avec laquelle on appuie sur le champignon? J'ai donc tout simplement décidé que les mesures étaient fausses. Avis confirmé sur Internet par un rapport de test. Pourtant, mesurer la tension artérielle devrait être simple.

Je suis très fier de mon nouvel appareil. Il mesure en haut du bras, en relation directe avec une application pour smartphone et affiche de superbes gra- phiques. Les valeurs sont plus stables, mais les écarts toujours présents. J'ai découvert que si je mesurais assis, une table élevée et une chaise basse pouvaient générer une différence de $20 \mathrm{~mm}$ par rapport à une chaise haute et à une table basse. N'importe quel professionnel vous le dira: toujours mesurer dans la même position, l'appareil à hauteur du cœur. Mesurer la tension artérielle est très simple... en principe.

Au grand dam de mon entourage, ce bijou peut mesurer à la demande trois fois d'affilée, avec des bruits de pompe et d'alertes. Les fabricants savent qu'il n'est pas si simple de mesurer la pression artérielle. Maintenant, je vois tout de suite quand les valeurs continuent de bondir dans les mêmes conditions (et elles le font régulièrement). Mais pourquoi? Problème d'appareil, de mesure ou de tension? Comme dirait Tucholsky: «Meine Probleme möchte ich haben.»

La mesure de la tension artérielle n'est pas aussi simple que ça. Evitons tout malentendu: il ne s'agit pas de ma tension artérielle, question d'ordre privée. Je me demande pourquoi cela paraît aussi facile avec les professionnels. Ils ne sont pourtant pas bêtes. Certes, ils ne font plus les erreurs des débutants. Mais comment gèrent-ils des valeurs/résultats aussi peu nets? Croient-ils vraiment aux mesures ponctuelles? Je suppose que la différence entre leur mesure et celle d'un patient comme moi, qui procède seul, réside dans le renoncement à savoir trop précisément dans certaines situations. Dans le quotidien professionnel, il ne faut pas trop réfléchir (on finirait par douter des valeurs limites officielles, mais c'est une autre histoire).

Existe-t-il seulement, dans le quotidien professionnel médical, des techniques pour écarter les facteurs d'incertitude sur les résultats particuliers (même pour les valeurs de laboratoire)? A part une considération de la moyenne à long terme? Un mélange d'expérience et de sensation, qui vous indique quand vous devez insister et comment? Je pense avoir moi-même développé quelque chose de la sorte pour la mesure de la tension, et je devine quand je dois recommencer.

Si vous voulez en savoir plus sur cette question, je vous recommande un article passionnant de Marianne Jossen, dans le numéro 13 du présent bulletin, parlant des décisions et de l'intuition dans la médecine.

Avec de telles techniques, la mesure de la pression artérielle redevient (plus) facile. N'importe quel professionnel vous le dira peut-être.

Eberhard Wolff * 